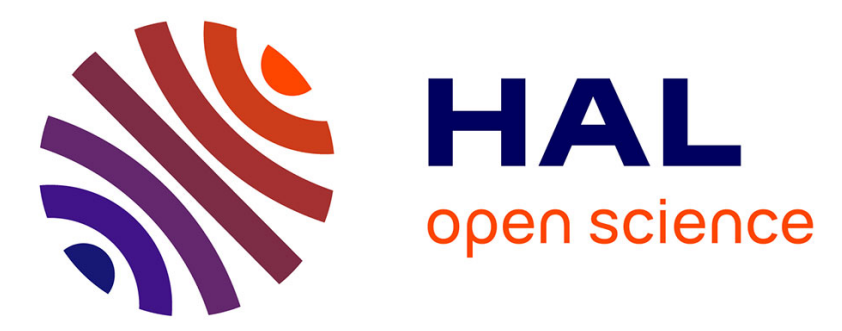

\title{
Kinetics of light-induced first-order phase transformation in molecular solids: $\mathrm{Fe}(\mathrm{btr}) 2(\mathrm{NCS}) 2 \mathrm{H} 2 \mathrm{O}$
}

Sébastien Pillet, Vincent Legrand, Mohamed Souhassou, Claude Lecomte

\section{To cite this version:}

Sébastien Pillet, Vincent Legrand, Mohamed Souhassou, Claude Lecomte. Kinetics of light-induced first-order phase transformation in molecular solids: Fe(btr)2(NCS)2 H2O. Physical Review B: Condensed Matter and Materials Physics (1998-2015), 2006, 74 (14), 10.1103/PhysRevB.74.140101 . hal01007006

\section{HAL Id: hal-01007006 https://hal.science/hal-01007006}

Submitted on 31 Oct 2018

HAL is a multi-disciplinary open access archive for the deposit and dissemination of scientific research documents, whether they are published or not. The documents may come from teaching and research institutions in France or abroad, or from public or private research centers.
L'archive ouverte pluridisciplinaire HAL, est destinée au dépôt et à la diffusion de documents scientifiques de niveau recherche, publiés ou non, émanant des établissements d'enseignement et de recherche français ou étrangers, des laboratoires publics ou privés. 


\title{
Kinetics of light-induced first-order phase transformation in molecular solids: $\mathrm{Fe}(\mathrm{btr})_{2}(\mathrm{NCS})_{2} \cdot \mathrm{H}_{2} \mathrm{O}$
}

\author{
S. Pillet, V. Legrand, M. Souhassou, and C. Lecomte \\ Laboratoire de Cristallographie et Modélisation des Matériaux Minéraux et Biologiques, LCM3B, UMR CNRS 7036, Université Henri \\ Poincaré, 54506 Vandoeuvre-les-Nancy, France
}

\begin{abstract}
The mechanism and kinetics of the thermally and light-induced spin transition of the highly cooperative spin crossover material $\mathrm{Fe}(\mathrm{btr})_{2}(\mathrm{NCS})_{2} \cdot \mathrm{H}_{2} \mathrm{O}$ (btr=4,4 /-bis-1,2,4-triazole) have been investigated by single crystal $\mathrm{x}$-ray diffraction techniques. The key role of like-spin domains is quantitatively analyzed through a nucleation, growing, and coarsening mechanism whose kinetics follow the Kolmogorov-Johnson-Mehl-Avrami model with low dimensional characteristics.
\end{abstract}

The study of structural reorganization in solids undergoing phase transformations is of fundamental importance; the nonequilibrium kinetics of such process has attracted intense research activities. Of prime interest are cases for which the phase transformation can be triggered by external stimuli (e.g., light, pressure). In this context, photoswitchable bistable molecular materials, among other spin crossover (SCO) complexes, are very promising systems exhibiting so called photoinduced phase transitions (PIPT). ${ }^{1}$ The nonlinearity of the light-induced processes suggests that cooperative interactions, originating from strong electron-lattice coupling, play a key role in the mechanism of PIPT. The dynamic behavior of these peculiar phase transformations is still under debate.

Evidences have been brought that universal process and scaling laws do exist for describing solid systems approaching equilibrium from a nonequilibrium state (e.g., thermally quenched metastable state). ${ }^{2}$ For instance, various crystallization kinetics follow the same transformation rate in reduced time units. For first-order phase transition, the transformation from the metastable phase is usually ascribed to germ nucleation of the thermodynamically stable phase followed by domain growing and coarsening. In the classical nucleation theory, nucleation and growing stages are temperature-dependent activated phenomena. Their kinetics are generally accounted for using the Kolmogorov-JohnsonMehl-Avrami (KJMA) model, ${ }^{2}$ which assumes a stochastic distribution of nucleation sites, a constant nucleation rate, and linear domain growing. In this model, the fraction of transformed phase is given by $X(t)=1-\exp \left\{-\left[k\left(t-\tau_{i}\right)\right]^{n}\right\} \cdot \tau_{i}$ is an incubation delay, $k$ is the rate constant related to a characteristic transformation time $k=1 / \tau_{\text {transf }}$, and $n$ is the Avrami exponent which reflects the degree of heterogeneity and process dimensionality. Many kinetics of phase transformations obey the KJMA rate law; it has been shown recently that this model also accounts quite well for light-induced transformations. ${ }^{3,4}$

Iron (II) SCO complexes exhibit a molecular bistability characterized by a high spin (HS) to low spin (LS) conversion on decreasing temperature, abrupt for strongly cooperative systems. LS to HS phase switching can be reversibly triggered and controlled using appropriate optical excitation (LIESST phenomenon). The participation of like-spin domains in the spin transition behavior has been recognized from the observation of Bragg peak splitting using $\mathrm{x}$-ray powder diffraction ${ }^{5}$ and single crystal $\mathrm{x}$-ray diffraction in the thermally ${ }^{6}$ and light-induced ${ }^{7}$ transition regimes. The structure and dynamics of like-spin domains are essential parameters in determining the use of these materials in data storage nanotechnology.

For weakly cooperative materials, the LS-HS lightinduced transformation and HS-LS relaxation kinetics follow monoexponential rates. In the case of high cooperativity, a characteristic sigmoidal curve is observed, which has been accounted for using the concept of autoacceleration. ${ }^{8} \mathrm{~A}$ mean field master equation has been proposed for describing all the stationary and dynamic behavior, including the light-induced ones. ${ }^{9}$ Spin transition kinetics are furthermore influenced by the degree of inhomogeneity. For instance, mechanical stressing of SCO powder samples ${ }^{5}$ or dispersion in polymer matrices ${ }^{10}$ result in deviation from purely exponential HS-LS relaxation rate. On the other hand, deviations from the KJMA kinetics are expected for fine grain microcrystalline powders for which growing is limited by the grain size. ${ }^{11}$ Based on these observations, single crystal investigation techniques are preferred.

We report in this paper on the analysis of the underlying mechanism and kinetics of the thermally and light-induced phase transitions of the archetype of highly cooperative SCO complex $\mathrm{Fe}(\mathrm{btr})_{2}(\mathrm{NCS})_{2} \cdot \mathrm{H}_{2} \mathrm{O}$ (btr=4,4 -bis-1,2,4-triazole) by temperature and time-dependent single crystal $\mathrm{x}$-ray diffraction technique using a charge-coupled device (CCD) detector. Owing to its very abrupt thermal spin transitions and large hysteresis loop, we expect that domain nucleation and growing might play the key role in the transition dynamics. The crystal structure of $\mathrm{Fe}(\mathrm{btr})_{2}(\mathrm{NCS})_{2} \cdot \mathrm{H}_{2} \mathrm{O}$ consists of bidimensional extended layers connected by van der Waals interactions and hydrogen bonds. ${ }^{12}$ This material is one of the only examples of two-dimensional (2D) polymeric SCO systems and thus deserves special attention, notably the influence of cooperativity on the spin transition kinetics. The spin transition properties ${ }^{13}$ exhibit a $21 \mathrm{~K}$ hysteresis and are given in Fig. 1. A nonlinear response to photoexcitation, as well as a light-induced instability, have been pointed out by fitting the evolution under continuous optical excitation of the fraction of molecular LS species $n_{\mathrm{LS}}(t)$ according to the macroscopic mean field master equation for doped $\mathrm{Fe}_{\mathrm{x}} M_{1-\mathrm{x}}(\mathrm{btr})_{2}(\mathrm{NCS})_{2} \cdot \mathrm{H}_{2} \mathrm{O}(M=\mathrm{Co}, \mathrm{Ni}, \mathrm{Zn})$ polycrystalline powders. ${ }^{14}$ 


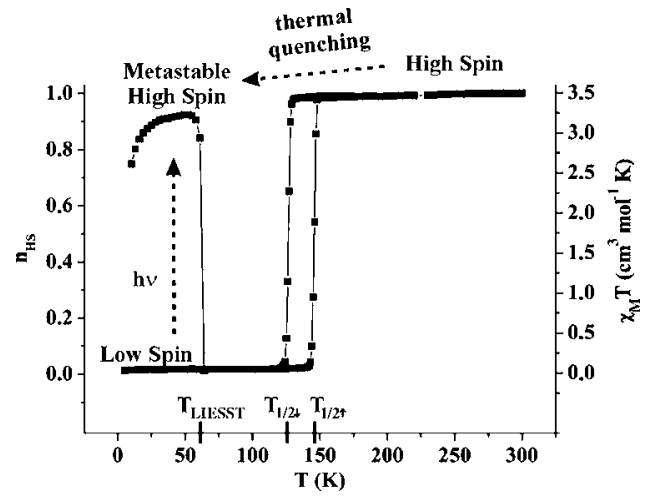

FIG. 1. Spin transition properties of $\mathrm{Fe}(\mathrm{btr})_{2}(\mathrm{NCS})_{2} \cdot \mathrm{H}_{2} \mathrm{O}$ from magnetic susceptibility measurements (Ref. 13).

Thermally induced transformation. Upon passing the thermally induced transition, the crystal lattice constants change abruptly, characteristic of first-order transition. Correlatively, the evolution of the x-ray diffraction pattern presents a splitting of the Bragg peaks, attributed to an inhomogeneous HS and LS phase coexistence [Fig. 2(a)], the phase transition mechanism is based on like-spin domain formation. ${ }^{6}$ Such behavior has also been reported for other cooperative SCO materials. On the contrary, during gradual transitions, a continuous displacement of the Bragg peak $2 \theta$ position occurs and linearly parallels $n_{\mathrm{HS}}(t) .{ }^{15}$ The isothermal evolution of the diffraction pattern of $\mathrm{Fe}(\mathrm{btr})_{2}(\mathrm{NCS})_{2} \cdot \mathrm{H}_{2} \mathrm{O}$ has also been recorded versus time following an overcooling to $\mathrm{T}$ $=117.2 \mathrm{~K}$, slightly below the thermal HS-LS transition $\left(T_{1 / 2 \downarrow}\right) .{ }^{6}$ The temporal resolution of the diffraction experiment $(\sim 10 \mathrm{~s})$ is limited by the CCD read out time and did not allow deriving any incubation time; accordingly, $\tau_{i}=0$ is assumed in the following. An incubation time of a few tens of seconds has been evidenced by optical absorption spectroscopy for the LS-HS photoconversion of $\left[\mathrm{Fe}(2-\mathrm{pic})_{3}\right] \mathrm{Cl}_{2} \cdot \mathrm{EtOH}^{16}{ }^{16}$ The integrated intensities of the Bragg peaks $I(t)$ follow a sigmoidal increasing or decreasing trend [Fig. 2(b)], characteristic of high cooperativity and autoaccelerated phenomenon in the Hauser formalism. ${ }^{8}$ This nonlinear intensity evolution can be rationalized using the following domain nucleation and growing arguments. By a slight overcooling below $T_{1 / 2 \downarrow}$, the system is brought in a region of the phase diagram where the LS state is thermodynamically stable. After an incubation time $\tau_{i}$, small LS clusters form via local fluctuations within the HS phase, corresponding to nucleation; LS molecular clusters of size greater than the critical size become stable nuclei. These small LS forming clusters should lead to diffuse scattering and a decrease of the Bragg peak intensity, corresponding to the HS structurally coherent matrix solely: $I_{\text {bragg }} \sim I_{\mathrm{HS}}$. The time scale of this phenomenon is certainly below our time resolution. Then, critical nuclei grow by incorporating neighboring molecules and gradually convert the HS phase to LS. During growing, domain sizes reach the intrinsic spatial resolution of our x-ray diffraction equipment, the transformed LS domains and the remaining HS matrix add incoherently to the total Bragg intensity signal $I_{\mathrm{Bragg}} \sim I_{\mathrm{HS}}+I_{\mathrm{LS}}$. For this configuration, a splitting of the Bragg peaks is observed corresponding to the HS matrix and the forming LS domains, respec-
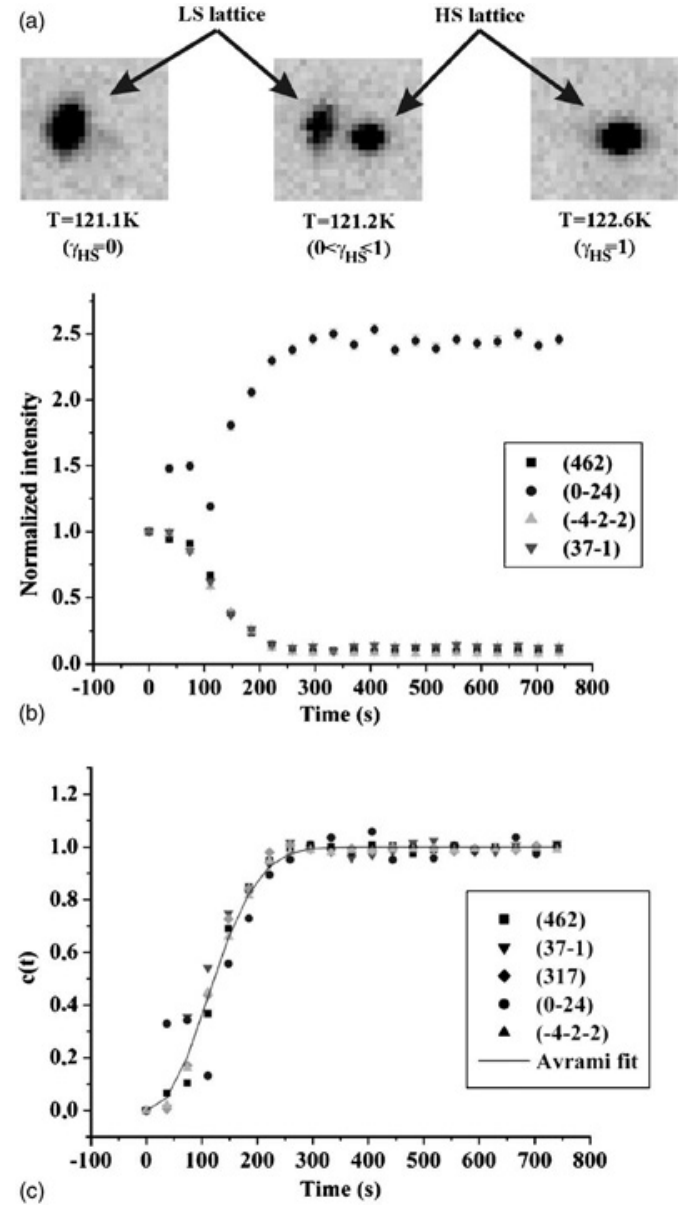

FIG. 2. Thermally induced transition in the cooling mode: (a) Temperature dependence of the (063) reflection x-ray diffraction pattern, (b) normalized integrated intensities $I(t) / I(t \rightarrow 0)$ for selected reflections $(h k l)$, (c) extent of phase transformation vs time $c(t)$.

tively. As the thermal HS-LS conversion proceeds, the positions in reciprocal space of the split HS and LS peaks remain unchanged ${ }^{6}$ the strain relaxation at the domain boundary is therefore a very local phenomenon and does not propagate to the whole crystal lattice: the nontransformed HS phase is structurally scarcely perturbed by the continuously transforming phase.

Based on the layer crystal structure of $\mathrm{Fe}(\mathrm{btr})_{2}(\mathrm{NCS})_{2} \cdot \mathrm{H}_{2} \mathrm{O}$, the phase transformation may proceed following two low dimensional mechanisms. In the first hypothesis, nucleation and growing first quickly develop in 2D followed by a $1 \mathrm{D}$ slow growing perpendicular to the layers. Alternatively, the rate limiting factor could be a slow 2D growing. The integrated intensities have been converted to a time dependent dimensionless extent of phase transformation $c(t)=[I(t)-I(t \rightarrow 0)] /[I(t \rightarrow \infty)-I(t \rightarrow 0)]$ [Fig. 2(c) $]$. The KJMA model accounts well for these observed kinetics, with a characteristic time $\tau_{\text {transf }}=145 \mathrm{~s}\left[k=0.0069(1) \mathrm{s}^{-1}\right]$ and an exponent $n$ between 2 and $3[n=2.2(1)]$, indicating either a deceleration of the nucleation process as more material is transformed into the stable phase (site saturation) or a low dimensional (2D) growing process. Surprisingly, common kinetics is observed for all Bragg reflections, irrespec- 


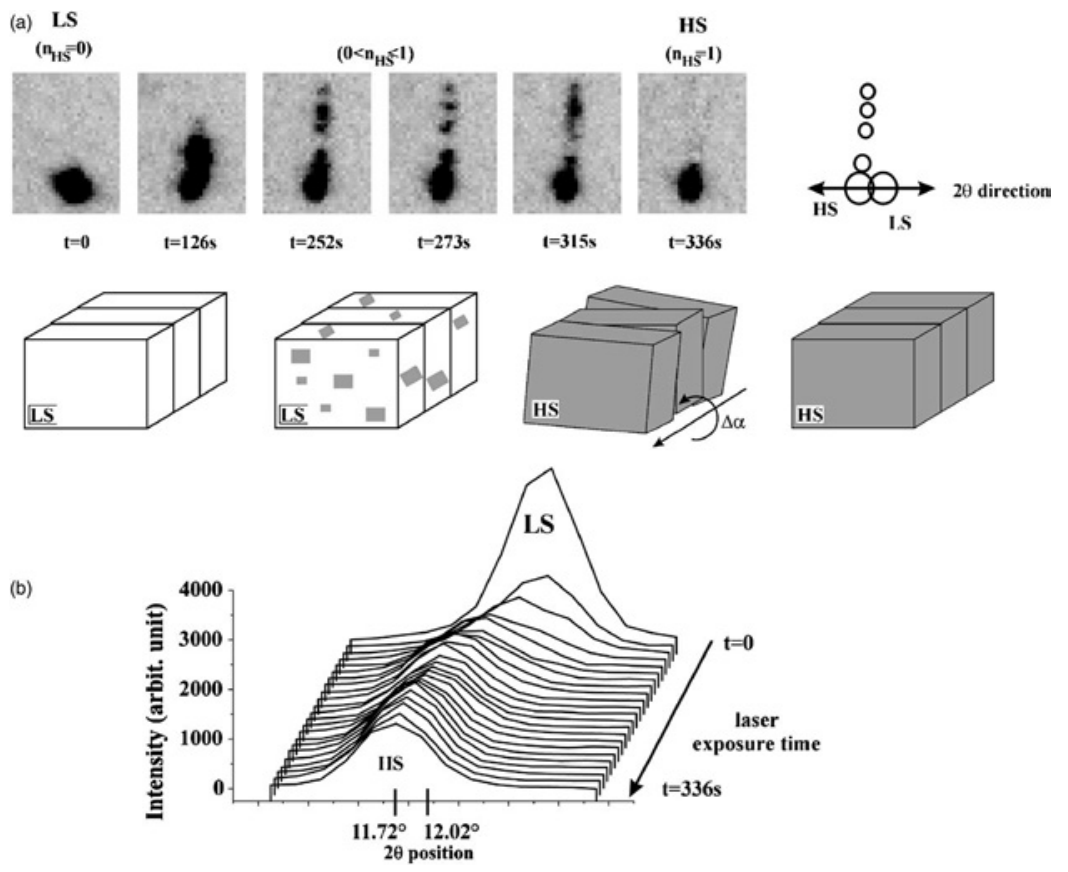

FIG. 3. Light-induced transformation: (a) Evolution of the diffraction pattern centered on the $\left(\begin{array}{lll}1 & 3 & -2\end{array}\right)$ reflection vs time, (b) evolution of the peak intensity along $2 \theta$ direction.

tive of their direction in reciprocal space. The mean field master equation ${ }^{9}$ is usually used to quantify such spin transition kinetics and accounts well for almost all magnetic or optical measurements. These techniques measure macroscopic quantities (optical density and magnetic susceptibility) directly (linearly in a first approximation) related to $n_{\mathrm{HS}}(t)$, whereas our diffraction experiment is sensible to long range ordered (structurally coherent) phases; accordingly, the extent of phase transformation $c(t)$ should not be confused with the extent of molecular species HS to LS conversion $\left[1-n_{\mathrm{HS}}(t)\right]$.

Light-induced transformation. The LS-HS photoconversion has been performed at $T=15 \mathrm{~K}$ using a cw Ar-Kr laser and followed by single crystal diffraction techniques. The laser excitation wavelength $(\lambda=488 \mathrm{~nm})$ corresponds to a characteristic absorption band of the LS ground state aside the maximum of absorption to ensure a high crystal penetration depth. Under such conditions, a homogeneous nucleation is expected. A laser power threshold has been observed below which no conversion occurred $\left(P_{\text {limit }}\right)$, as also discussed by Ichiyanagi et al. for $\mathrm{Fe}(\mathrm{PM}-\mathrm{Bia})_{2}(\mathrm{NCS})_{2} \cdot{ }^{17}$ This threshold, usually attributed to a competition between the LS-HS photoexcitation and HS-LS relaxation, is reproduced within the mean field master equation approach ${ }^{18}$ or electron lattice coupling models including long range interactions. ${ }^{19}$ For $\mathrm{Fe}(\mathrm{btr})_{2}(\mathrm{NCS})_{2} \cdot \mathrm{H}_{2} \mathrm{O}$, the HS-LS relaxation rate is almost negligible at $T=15 \mathrm{~K}$, the threshold should therefore result from cooperative elastic interactions solely. The eventual dependence of the transformation kinetics with laser power $P\left[P_{\text {limit }}\right.$ and $\left.k(P)\right]$ needs further investigation.

The LS-HS light-induced transition exhibits additional specific features with respect to the thermally induced one. The evolution of the diffraction pattern can be separated into consecutive regimes with different characteristic times [Fig. 3(a)]. First the molecular LS-HS conversion occurs as evidenced by the $2 \theta$ displacement of the Bragg peak position
[Fig. 3(b)], along with a splitting of the LS and HS Bragg peaks over several well separated positions [e.g., Fig. 3(a) at $t=273 \mathrm{~s}]$. This step corresponds to the nucleation and growing process. At the end of domain growing, the single crystal consists of HS polymicrodomains with identical HS lattice constants but slightly disoriented with respect to each other (Fig. 3, schematic picture). Based on the layer crystal structure, it is more likely that the disorientation occurs perpendicular to the crystallographic $a$ axis. Remarkably, in the late stage of the transition (from $t=315 \mathrm{~s}$ to $t=336 \mathrm{~s}$ ), the separated HS Bragg peaks of lowest intensity coalesce towards the major peak; no further evolution is observed above $336 \mathrm{~s}$. Final equilibrium is reached by the shrinking of the smallest size domains and a consequent growth of the major ones. Domain coarsening is therefore evidenced in spin transition materials and molecular crystals, probably due the particular layer structure and therefore highly 2D cooperativity of $\mathrm{Fe}(\mathrm{btr})_{2}(\mathrm{NCS})_{2} \cdot \mathrm{H}_{2} \mathrm{O}$. The driving force for domain coarsening is a decrease of the interdomain boundary elastic energy. The crystal structure solved for the phototransformed phase $(t>336 \mathrm{~s})$ corresponds to a pure HS state $\left(n_{\mathrm{HS}}=1\right)$ with Fe-N coordination bond distances similar within $0.01 \AA$ to the room temperature HS ones. ${ }^{13}$ We do not observe any saturation effect $\left[n_{\mathrm{HS}}(t \rightarrow \infty)<1\right]$ which could be attributed to a bleaching of the nucleation and a saturation of the growing process due to strong spontaneous strain induced by the large LS-HS cell volume misfit. In some special cases, the absence of observed peak splitting even for highly cooperative systems like $\left[\mathrm{Fe}(\mathrm{ptz})_{6}\right]\left(\mathrm{BF}_{4}\right)_{2}$, has been attributed to a hindrance of the domain growing process, ${ }^{20}$ contrary to $\mathrm{Fe}(\mathrm{btr})_{2}(\mathrm{NCS})_{2} \cdot \mathrm{H}_{2} \mathrm{O}$.

The evolution of the $2 \theta$ peak profile upon laser exposure has been quantitatively analyzed [Fig. 3(b)]. The peak profiles at $t=0 \mathrm{~s}\left(n_{\mathrm{HS}}=0\right)$ and $t=336 \mathrm{~s}\left(n_{\mathrm{HS}}=1\right)$ have been fitted to single Gaussian components centered at $2 \theta_{\mathrm{LS}}=12.02^{\circ}$ and $2 \theta_{\mathrm{HS}}=11.72^{\circ}$, respectively. In the intermediate situations $\left(0<n_{\mathrm{HS}}<1\right)$, the peak profiles have been described as a 


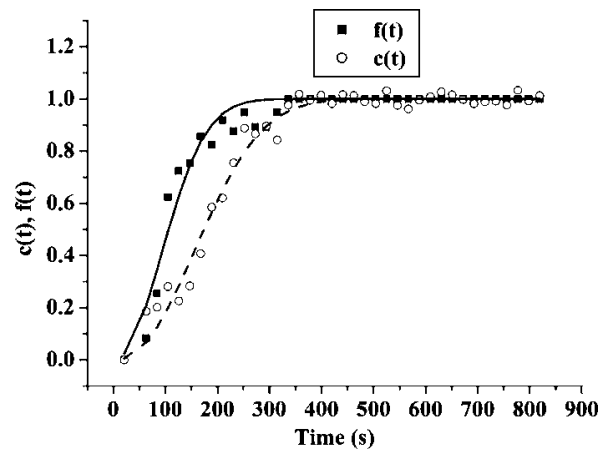

FIG. 4. Light-induced transformation: Avrami fit of $c(t)$ and $f(t)$ for the (1 $3-2)$ Bragg reflection.

weighted superposition of the HS and LS components; the weights being $P_{\mathrm{HS}}(t)$ and $P_{\mathrm{LS}}(t)$, respectively. Based on this, the transformation kinetics can be investigated in two ways, using the $2 \theta$ peak position or corresponding integrated intensity. The ratio $f(t)=P_{\mathrm{HS}}(t) /\left[P_{\mathrm{HS}}(t)+P_{\mathrm{LS}}(t)\right]$ exhibits a clear nonlinear sigmoidal rate versus laser exposure time (Fig. 4). A least-squares fit to the KJMA model leads to $n=2.1(1)$ and $\tau_{\text {transf }}=127 \mathrm{~s}\left[k=0.0079(2) \mathrm{s}^{-1}\right]$. On the other hand, the overall peak intensity gives information on the long range spatial coherence of the HS and LS domains. The evolution of the overall normalized peak intensity $c(t)=[I(t)-I(t \rightarrow 0)] /[I(t \rightarrow \infty)-I(t \rightarrow 0)]$ has also been fitted to the KJMA model (Fig. 4), leading to an Avrami exponent of $n=2.1(2)$, similar to those derived from the $2 \theta$ peak position. However, the phase transformation characteristic time of $\tau_{\text {transf }}=205 \mathrm{~s}\left[k=0.00488(1) \mathrm{s}^{-1}\right]$ is clearly longer. We argue that this is due to the late stage of the transformation and domain coarsening. When the LS to HS molecular conversion is almost achieved and the crystal sample consisting of HS polydomains, the $2 \theta$ peak position corresponds to $2 \theta_{\mathrm{HS}}=11.72^{\circ}[f(t) \sim 1]$. On the other hand, the incoherent scattering due to the intra-domain boundary and small size domains leads to a decrease of the Bragg intensity $[c(t)<1]$. A full recovery of the final intensity occurs after the domain coarsening is achieved, that is to say when the crystal lattice has reached an equilibrium state and a single orientation.
The photomagnetic properties of $\mathrm{Fe}(\mathrm{btr})_{2}(\mathrm{NCS})_{2} \cdot \mathrm{H}_{2} \mathrm{O}$ have been analyzed and a temperature $T_{\text {LIESST }}=61 \mathrm{~K}$ has been quantified. ${ }^{13}$ Based on this $T_{\text {LIESST }}$ relaxation temperature, a single crystal prepared in the HS metastable state at $15 \mathrm{~K}$ by thermal quenching has been warmed up to $50 \mathrm{~K}$, below $T_{\text {LIESST }}$, and the x-ray diffraction pattern recorded versus time along the relaxation. This isothermal phase transition exhibits the splitting of the Bragg peak (HS and LS phase coexistence) but does not show any evidence for domain coarsening contrary to the light-induced transformation. It is concluded that this latter undergoes a specific mechanism. It has been recently suggested that, close to the critical temperature of the light-induced thermal hysteresis (LITH), light-induced organization of like-spin domains could occur. $^{21}$ We could suppose that for $\mathrm{Fe}(\mathrm{btr})_{2}(\mathrm{NCS})_{2} \cdot \mathrm{H}_{2} \mathrm{O}$, domain coarsening is induced or at least enhanced by light excitation, even though the experiment temperature $(15 \mathrm{~K})$ is well below the critical temperature $(61 \mathrm{~K})$. By comparing the linewidth of $\mathrm{x}$-ray powder diffraction pattern during light-induced and thermal transition of a Prussian blue analog, a larger domain size has been evidenced for the photoinduced phase, enlightening even more the particular light induced transformation processes. ${ }^{22}$

Our findings suggest that the first-order thermally and light-induced transitions of the SCO material $\mathrm{Fe}(\text { btr })_{2}(\mathrm{NCS})_{2} \cdot \mathrm{H}_{2} \mathrm{O}$ follow the Kolmogorov-Johnson-MehlAvrami model with characteristic exponent $n$ in the range 2.0-2.5. The mechanism is of low dimensionality, in agreement with the layer crystal structure. However, no evidences have been found for a dual growing process with different characteristic time, namely 2D-layer development followed by interlayer conversion. The late time of the light-induced transition corresponds to domain coarsening of the completely converted HS state, which could be related to lightinduced organization.

This work was financially supported by the European Network of Excellence MAGMANet (Grant No. FP6-515767-2), the Université Henri Poincaré, and the CNRS. V.L. is indebted to the Ministère de l'Education Nationale, de l'Enseignement Supérieur et de la Recherche for support. We thank J. F. Létard for his help during magnetic measurements.
${ }^{1}$ Relaxations of Excited States and Photoinduced Phase Transitions, edited by K. Nasu (Springer-Verlag, Berlin, 1997).

${ }^{2}$ A. N. Kolmogorov, Bull. Acad. Sci. USSR, Phys. Ser. (Engl. Transl.) 3, 355 (1937); W. A. Johnson and R. F. Mehl, Trans. Am. Inst. Min., Metall. Pet. Eng. 135, 416 (1939); M. Avrami, J. Chem. Phys. 7, 1103 (1939); 8, 212 (1940).

${ }^{3}$ M. Bertmer et al., J. Phys. Chem. B 110, 6270 (2006).

${ }^{4}$ X. Liu et al., J. Phys. Soc. Jpn. 69, 1267 (2000).

${ }^{5}$ E. W. Müller et al., J. Chem. Phys. 79, 1439 (1983).

${ }^{6}$ S. Pillet et al., Eur. Phys. J. B 38, 541 (2004).

${ }^{7}$ N. Huby et al., Phys. Rev. B 69, 020101(R) (2004).

${ }^{8}$ A. Hauser et al., Inorg. Chem. 25, 4245 (1986).

${ }^{9}$ A. Desaix et al., Eur. Phys. J. B 6, 183 (1998); F. Varret et al., Mol. Cryst. Liq. Cryst. Sci. Technol., Sect. A 334, 1273 (1999).

${ }^{10}$ A. Hauser et al., Chem. Phys. Lett. 152, 468 (1988).
${ }^{11}$ P. T. Cardew et al., J. Chem. Soc., Faraday Trans. 2 80, 659 (1984).

${ }^{12}$ W. Vreugdenhil et al., Polyhedron 9, 2971 (1990).

${ }^{13}$ V. Legrand, Ph.D. thesis, University Henri Poincaré, Nancy I (France), 2005.

${ }^{14}$ C. Enachescu et al., J. Phys. Chem. Solids 62, 1409 (2001).

${ }^{15}$ E. Meissner et al., Chem. Phys. Lett. 95, 163 (1983).

${ }^{16}$ Y. Ogawa et al., Phys. Rev. Lett. 84, 3181 (2000).

${ }^{17}$ K. Ichiyanagi et al., Phys. Rev. B 73, 060408(R) (2006).

${ }^{18} \mathrm{~F}$. Varret et al., Polyhedron 24, 2857 (2005).

${ }^{19}$ K. Koshino and T. Ogawa, J. Phys. Soc. Jpn. 68, 2164 (1999).

${ }^{20}$ A. Goujon et al., Phys. Rev. B 73, 104413 (2006).

${ }^{21} \mathrm{~F}$. Varret et al. (private communication).

${ }^{22}$ M. Hanawa et al., J. Phys. Soc. Jpn. 72, 987 (2003). 